\section{IJ§ER}

ISSN: 2149-5939
International Journal of Social Sciences and Education Research

Online, http://dergipark.gov.tr/ijsser

Volume: 3(4), 2017

\title{
The impact of infographic animation videos on data visualization
}

\author{
Semih Delil ${ }^{1}$
}

Received Date: 11 / 03 / 2017

Accepted Date: 14/ 06 / 2017

\begin{abstract}
Visualized information has not lost its significance since from time that we were living in caves. Visual support to information which is meant to be explained, or its totally visual presentation, facilitates its reaching to the target audience. Information transfer throughout images is a long process, ranging from pictograms to graphics and even to motion graphics. We frequently meet with these images in our everyday lives. These infographics, which facilitate our everyday life, provide us a faster understanding of the environment we are living in or the information we read.
\end{abstract}

Keywords: Infographics, animation, data visualization, data

\section{Introduction}

Information design is not a new phenomenon. Ever since the old cave drawings people are using graphics to describe information (Lankow 2012). We face more and more data every day with the development of technology. This growing mass of data has brought the desire to make sense of this information (Knaflic 2015). Nowadays sending a visual message is an important communication problem. We are becoming pullers of information rather than accepting that which is pushed to us (Bellato 2013).

Printed versions of infographics are inadequate when the data which will be transferred increased. In this context, the importance of transferring data to the audience quickly and effectively is increasing. Short information, tables, figures and values are the most basic components used to visualize the data. However, when these components are intended to emphasize the contents of the data which will be transferred to the audience, transferring information through a fixed medium cannot create the desired effect. Therefore, the content to be transferred must be remarkable, attractive and memorable.

\section{What is infographic?}

\subsection{Infographic}

An infographic is a type of picture that blends data with design, helps individuals and organizations in order to transfer their messages to their audience. (Smiciklas, 2012) Infographic is not just the arrangement of images. At the same time, infographics contain carefully arranged forms of typographic elements. Design for information offers basic learning methods and graphics for visual presentation of information (Meirelles, 2013) Infographic is a group of graphs Research Assistant, Baskent University Faculty of Communication, Ankara/TURKEY,
semihd@baskent.edu.tr 
Delil, S (2017). The impact of infographic animation videos on data visualization. International Journal of Social Sciences and Education Research, 3 (4), 1178-1183.

that visualize a stack of information or infographic information. The infographic standard conveys a more creative language rather than a plain writing style. In these days, infographics are often confronted in the media, in printed work, on road signs and in manuals.

Figure 1. Deciding how to explain

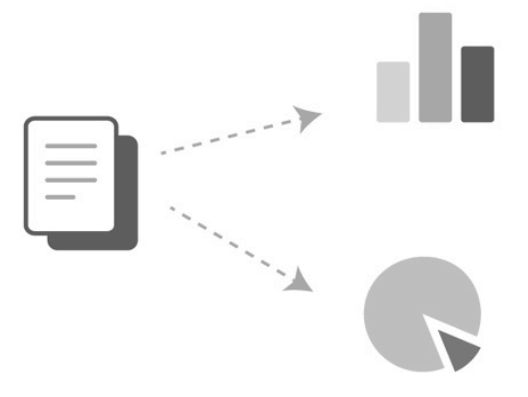

\subsection{Infographic videos}

Videos have been an effective medium for information transfer in today's world. In web searches, videos are preferred 53 times more than a normal website for information (Kalinia 2014).

In infographic videos, units such as tables, measurements, and date are combined together. The definition of information visualization animations in a broad sense can be done as follows: infographic animations are called to animate audio visual items in the heap of information which is needed to be conveyed in a correct and curious way by taking the elements of communication, visual communication and graphic design into account.

Figure 2. Combining data

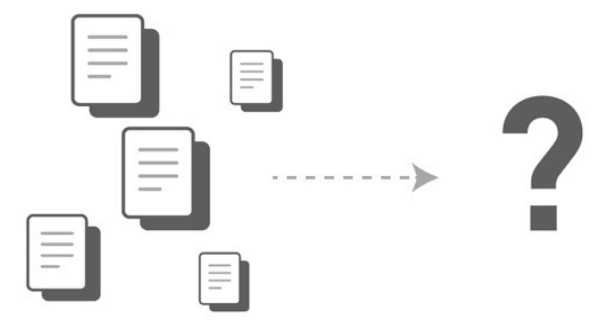

Information visualization is a more effective method for transferring information to people rather than the audio or written media. Animation of these static visuals with animation techniques increases the effect of information transfer.

It will be more accurate to make the information transfer with the videos today since the videos are preferred rather than the visual and written councils.

\subsection{Rules should be considered in infographic video design}

Designing an infographic video is different than designing a brochure, website, or a catalogue. While establishing an information graph, attention should be given to the correct communication and communication design rules with the target audience, rather than the standard graphic design rules. 
Delil, S (2017). The impact of infographic animation videos on data visualization. International Journal of Social Sciences and Education Research, 3 (4), 1178-1183.

Fig.3. From storyboard to movie

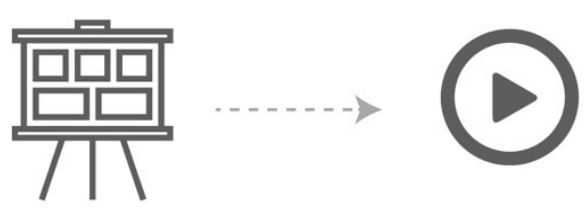

An infographic should be shown not be told. In an infographic, the information should be expressed in visual terms (such as figures, graphics, maps etc.) first. The same rule should be applied to infographic animation videos. When the information that will be delivered to the target group is too much and the content of the program is limited, the animation videos are chosen.

When it is desired to transfer a certain amount of information to the target group with infographic animation, the visualization of the information should be directly proportional to the perception process of the target group.

\section{Getting started with infographic animation design}

\subsection{Drawing process}

An important part of information design is information graphics and visualization (Cairo 2012) Our eyes tend to watch very often. They prefer to look instead of reading. If our brains had a preference, they would most likely prefer visual information. (Bellato 2013)

Visualizing information does not mean creating standard bar or circle graphs. Designing infographic animation in the same way only means animating this bar or hoop graphics.

The most important rule in the infographic animation design is that each data is described with a unique language and graphics. The reason for this situation is that repetitive visuals are not very successful in remembrance. With this way, each originally designed scene will not bore the viewer and wonder them at every step.

\subsection{Typography}

\subsubsection{The Importance of Typography}

Written language is a material that conveys the message of the cultures and is important for education at the same time. Nowadays, the increasing use of computers is accompanied by written text and moving images. In this context, the use of typography needs to be done effectively in order to make readers to be able to read on the screens correctly. In order to be able to read the text on the screen, there should be an effective relationship with the media in which the text is displayed. Moreover, readability is also related to the nature of the writing, to the story, and to the expression of the story (Ambrose \& Harris, 2005).

The letters form the words when they come together. The characters in this formation play a major role in the reading. Articles are printed or displayed letters, words and phrases with their own style and dimensions. With the rapid development of technology, users started to spend 
Delil, S (2017). The impact of infographic animation videos on data visualization. International Journal of Social Sciences and Education Research, 3 (4), 1178-1183.

much time with computers, especially with computer-based online sources, in many places, (Ferrari \& Short, 2002).

Typography is one of the indispensable pieces of a design work. But when it comes to solving the problem of transferring information, it is an influence that should be consulted as little as possible. Overuse typography in an infographic video causes distraction for the viewer.

\subsubsection{Typography and colour}

Colours are an important issue in infographic typography. Distraction is very frequent when someone reads on the screen. When reading and visual impact is encountered, the importance of well-designed typography increases. In this case, basic colour usage rules can be effective. For instance, colours such as green and red, white and yellow should not be used together.

Infographic animations often contain moving typography. In these moving typographies, the use of contrasting colours can be difficult for tracking if the contrasts are effective. If contrast will be used on the effect and you have to use two colours, you need to use contrasting colours which are neither very contrasting nor very faint (Sala 2014).

Figure 4. Tints of typography

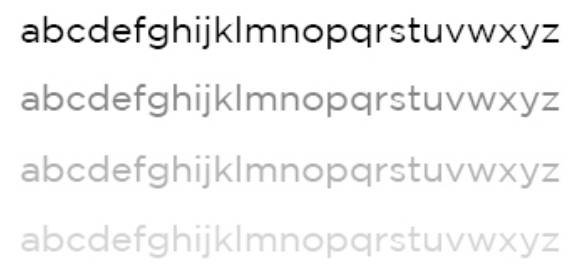

\subsection{Organization and storyboard}

Storyboards are the first element of narrative. Creation of this visual is the beginning of the plan. Generally, It is more effective to tell a situation visually rather than in written form. Thanks to the storyboards, the design process is more organized and faster. With this way, elements such as timing, movement, attention, colour and expression are better planned.

For instance, animation production process is long and difficult. Making the organization with storyboards allows the organization to work correctly. The correct operation of the organization will make the animation production process faster. Storyboards are used not only in planning but also in the process of emerging new ideas.

Information that is transferred to infographic videos is often more than a regular infographic. In infographic animations, the area is restricted according to a normal infographic. Therefore, the storyboards should describe each piece of information in staggered scenes.

\section{The Influence of infographic videos}

It is observed that watching videos are more preferred than reading a text in today's world. In this context, stationary infographic studies in data transmission are not very influential due to the area covered. Infographics are often remarkable and this makes them easier to share (Kukral 
Delil, S (2017). The impact of infographic animation videos on data visualization. International Journal of Social Sciences and Education Research, 3 (4), 1178-1183.

2012). The choice of moving infographic videos is increasing day by day in order to make it easier to share in electronic media and to reach more audience.

Figure 5. Putting information together

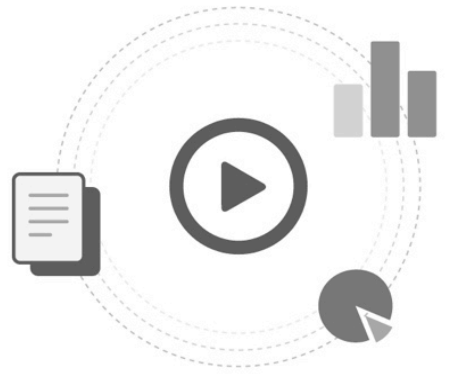

\section{Conclusions}

Transferring information is an important issue. The amount of information that we are exposed to increases day by day. This is important if you need to benefit from the piles of information quickly. Thanks to technology, we now prefer videos for information exchange. However, a visual and auditory video can sometimes lose its effect when the accessed information is based on data. In this case, information infographics offer a more memorable and easier way of communication. But the stability of infographics is ineffective if the information is complex. Infographics that help us to understand complex data easily can lose their impact if the amount of data increases. In this case, infographic videos are introduced. Animated infographic videos both transfer higher amounts of data with faster and make it more memorable.

Animated and descriptive infographic animations are very effective for narration. If the content that will be told is large in terms of data, the solution is to prefer moving graphics. Infographic animation should not only increase the relevance of the construction of videos. The main aim of infographic videos is to transform the concept into visual data throughout visuals. The infographics prepared for this purpose are informative and they support the effective and correct transmission of information. This process is not easy but takes a long time. The aim of the animation in this process is not to present only to the target audience. At the same time, these videos should be designed to make the target data visually clear.

The information that will be conveyed can be ineffective with visual and auditory expressions. The style of expression, which is far from being static and is animated with moving graphics is more memorable. This form of expression is impressive and can be a faster data transmitter for viewers. In this case, transferring information with moving graphics provides a positive effect in terms of time and effect in the process of obtaining information.

\section{References}

Ambrose, G. \& Harris, P. (2006). Basics design 03: Typography. Lousanne. Switzerland: AVA Publishing.

Bellato N. (2013). Infographics: A visual link to learning. Retrieved from: http://elearnmag.acm.org/archive.cfm?aid=2556269 
Delil, S (2017). The impact of infographic animation videos on data visualization. International Journal of Social Sciences and Education Research, 3 (4), 1178-1183.

Cairo, A. (2012). The Functional Art: An Introduction to Information Graphics and Visualization. California: New Riders.

Ferrari, T. \& Short, C. (2002). Legibility and readability. Retrieved from: http://bigital.com/english/files/2008/04/web_legibility_readability.pdf

Kalinia A. (2014). Video Is the New Face of Content Marketing, Infographic. Retrieved from: http://www.business2community.com/infographics/video-new-face-content-marketing-infographic0799484

Knaflic, C. N. (2015). Storytelling with Data: A Data Visualization Guide for Business Professionals. New Jersey: Wiley.

Kukral J. (2012). Explainer Videos Are the New Infographics. Retrieved from: http://www.huffingtonpost.com/jim-kukral/social-media-marketing-videos-_b_1895514.html

Lankow, J. (2012). Infographics: The Power of Visual Storytelling. New Jersey: Wiley.

Meirelles I. (2013). Design for Information: An Introduction to the Histories, Theories, and Best Practices Behind Effective Information Visualizations. Beverly MA: Rockport Publishers

Sala S. (2014). Typography Cheat Sheet: The 6 Big Mistakes to Avoid. Retrieved from: http://www.sitepoint.com/typography-cheat-sheet/

Smiciklas M. (2012). The Power of Infographics: Using Pictures to Communicate and Connect With Your Audiences Indiana: Pearson Education. 